\title{
Rate Regions of a Two-Way Gaussian Relay Channel
}

\author{
Jianquan Liu, Meixia Tao and Youyun Xu \\ Department of Electronic Engineering \\ Shanghai Jiao Tong University, Shanghai, 200240, China \\ Email: \{jianquanliu, mxtao\}@sjtu.edu.cn
}

\begin{abstract}
We study two-way communications over a threenode Gaussian relay channel. Several practical relaying strategies are considered, including bitwise XOR-based decode-andforward (DF), superposition-based DF, and amplify-and-forward (AF). For each considered two-way relay scheme, we show that its achievable rate pair is governed by a set of linear inequalities. As a result, we obtain explicit, analytical expressions as well as geometrical shapes of the rate regions for each scheme. Our analytical results provide a convenient and efficient way to evaluate the performance of two-way relaying at any channel conditions. Numerical examples are also presented to demonstrate and compare the rate regions and maximum sumrates of the considered schemes at different relay locations.
\end{abstract}

\section{INTRODUCTION}

Recently, the concept of network coding [1] has been applied in cooperative networks to further improve the system spectral efficiency. An exemplary scenario is the two-way relaying, proposed by [2]-[8]. In a two-way relay channel, two source nodes communicate with each other with the help of a relay node. The relay node combines the bidirectional information flows, while each desired destination performs self-interference cancelation to detect the desired signal. Compared with traditional one-way relaying, two-way relaying can increase the spectral efficiency significantly.

From information theoretic perspective, two-way communication using a relay node can be regarded as a two-phase communication, the multiple access phase, where the two source nodes send information to the relay simultaneously or sequentially, and the broadcast phase, where the relay broadcasts the combined signal to the two destinations. Oechtering, et al, determine the capacity region of the broadcast phase in terms of the maximal probability of error [9]. The achievable rate region of a general two-way relay channel is studied by Xie using random binning [10]. On the other hand, authors in [7], [8], [11]-[14] consider more practical relay strategies over Gaussian channels. In specific, the expressions of rate pairs for AF relaying are obtained in [7], [8]. Authors in [8], [13] also obtained the rate pair expressions for superposition based DF relaying, where the relay node simply applies linear combining on the two decoded and re-modulated signals. The achievable sum-rate for bit-level XOR based DF is analyzed in [11]. Therein, the optimal time control with the objective

This work is supported by Shanghai Educational Development Foundation (No. 2008CG14) and by Natural Science Foundation of Shanghai (No. 08ZR1410400). of maximizing the sum-rate is studied. It is worth mentioning that the asymmetry in both packet size and channel gain of the two transmitting nodes is considered in [11]. More recently, Liu and Xue characterize the achievable rate region for one particular XOR based DF with the absence of direct link [12]. Note that Wyrembelski, et al, compared the achievable rate regions of 2-step XOR-based DF and superposition-based DF schemes in [14], but the authors did not consider the direct link and different length of packets received from different source node. Given the works mentioned above, it is still yet to know which relay scheme is the best, and there is a lack of a unified metric to compare different schemes.

In this paper, we provide an analytical study of rate regions of the various two-way relay schemes over Gaussian channels, including XOR-based DF, superposition-based DF, and with or without direct link. Explicit, analytical expressions as well as geometrical shapes of the rate regions achieved by the schemes are obtained. Using some numerical examples, we demonstrate and compare the rate regions and maximum sum-rates of these schemes at different relay locations. Some useful findings are observed.

\section{SYSTEM MODEL}

\section{A. Channel model}

We consider a two-way relay channel which consists of two source nodes and a relay node. The source nodes, denoted as 0 and 2, wish to exchange information through the relay node, denoted as 1 . We assume that all the nodes are halfduplex. The channel on each communication link is assumed to be Gaussian with fixed channel gain. The signal-to-noise ratio (SNR) from node $i$ to node $j$ is denoted as $\gamma_{i j}$, for $i, j \in$ $\{0,1,2\}$. Note that $\gamma_{i j}$ may not be equal to $\gamma_{j i}$ as the transmit power of node $i$ can be different from node $j$. The channel capacity of the link from node $i$ to node $j$ is denoted as $C_{i j} \triangleq$ $C\left(\gamma_{i j}\right)=\log _{2}\left(1+\gamma_{i j}\right)$. The sum-rate capacity of the multiple access channel (MAC) from nodes 0 and 2 to node 1 is denoted as $C_{\mathrm{m}} \triangleq C\left(\gamma_{01}+\gamma_{21}\right)=\log _{2}\left(1+\gamma_{01}+\gamma_{21}\right)$.

\section{B. Two-way relay schemes}

Similar to [11], we classify the existing two-way relay schemes into 3-step schemes and 2-step schemes according to the number of phases to finish one round of information exchange. 
1) 3-step schemes: In the first step, node 0 transmits and node 1 and 2 listen (node 2 listens only when direct link is available). In the second step, node 2 transmits and node 1 and 0 listen (node 0 listens only when direct link is available). In the third step, node 1 transmits and node 0 and 2 listen. The relay node 1 applies the DF strategy, which can be further divided into XOR-based DF and superposition-based DF. Let $\mathbf{b}_{0}$ and $\mathbf{b}_{2}$ denote the information bit sequences sent from the two source nodes 0 and 2, respectively. In XOR-based DF, the relay node performs bit-level XOR operation on the two sequences, resulting in a new bit sequence $\mathbf{b}_{0} \oplus \mathbf{b}_{2}$. The new sequence is then encoded and transmitted simultaneously to the two desired destinations. In superposition-based DF, the relay encodes the two bit sequences separately into complexvalued baseband signals $\mathbf{x}_{0}$ and $\mathbf{x}_{2}$ and then superimposes them together as $\sqrt{\theta} \mathbf{x}_{0}+\sqrt{1-\theta} \mathbf{x}_{2}$, where $\theta$ is a power allocation coefficient. The signal $\sqrt{\theta} \mathbf{x}_{0}+\sqrt{1-\theta} \mathbf{x}_{2}$ is then broadcasted directly. When there is no direct link, we refer to the above two schemes as 3-step with no direct link by XOR (3S-ND-XOR) and 3-step with no direct link by superposition (3S-ND-SUP). Similarly, when direct link is present, we refer to them as 3-step with direct link by XOR (3S-WD-XOR) and 3 -step with direct link by superposition (3S-WD-SUP).

2) 2-step schemes: In the first step, nodes 0 and 2 transmit simultaneously while node 1 listens. In the second step, node 1 transmits and nodes 0 and 2 listen. When DF strategy is adopted at the relay, the XOR-based and superposition-based $\mathrm{DF}$, similar to those in 3-step schemes, can be performed, and we refer to these schemes as 2-step by XOR (2S-XOR) and 2-step by superposition (2S-SUP). When AF strategy is adopted, the relay simply amplifies the received superimposed signal from the two source nodes and forwards it to the two receivers. We name this scheme as $2 \mathrm{~S}-\mathrm{AF}$ [8], [11].

\section{ANALYSIS OF RATE REGION}

Let $R_{0}$ and $R_{2}$ denote the data rates of the information flows $0 \rightarrow 2$ and $2 \rightarrow 0$, respectively, in the considered twoway communications. In this section, we derive the rate region $\left(R_{0}, R_{2}\right)$ of the aforementioned relay schemes.

\section{A. Achievable rate region for XOR-based DF}

Without loss of generality, we assume $\gamma_{10} \geq \gamma_{12}$ and thus $C_{10} \geq C_{12}$ throughout this subsection.

Theorem 3.1 (3S-WD-XOR): The rate region for 3S-WD$\mathrm{XOR}$ is the quadrilateral formed by the origin, point $\mathrm{A}$ with coordinate $\left(0,\left(1 / C_{21}+1 / C_{10}-C_{20} /\left(C_{21} C_{10}\right)\right)^{-1}\right)$, B with $\left(\left(1 / C_{01}+\left(C_{01}-C_{02}\right) /\left(C_{01} C_{12}\right)+\left(C_{01}-C_{02}\right) /\left(C_{01}\left(C_{21}-\right.\right.\right.\right.$ $\left.\left.\left.C_{20}\right)\right)\right)^{-1},\left(\left(1 / C_{21}+\left(C_{21}-C_{20}\right) /\left(C_{21} C_{12}\right)+\left(C_{21}-\right.\right.\right.$ $\left.\left.\left.C_{20}\right) /\left(C_{21}\left(C_{01}-C_{02}\right)\right)\right)^{-1}\right)$, and $\mathrm{C}$ with $\left(\left(1 / C_{01}+1 / C_{12}-\right.\right.$ $\left.\left.C_{02} /\left(C_{01} C_{12}\right)\right)^{-1}, 0\right)$, as sketched in Fig. 1(a).

Proof: Without loss of generality, the total time for one round of information exchange is fixed and normalized to 1 . The time fraction spent on each step is varying. Let $D_{0}$ and $D_{2}$ denote the packets to be communicated by the two source nodes, and their packet length in information bits be denoted as $\left|D_{0}\right|$ and $\left|D_{2}\right|$. In the first two steps, it is obvious that

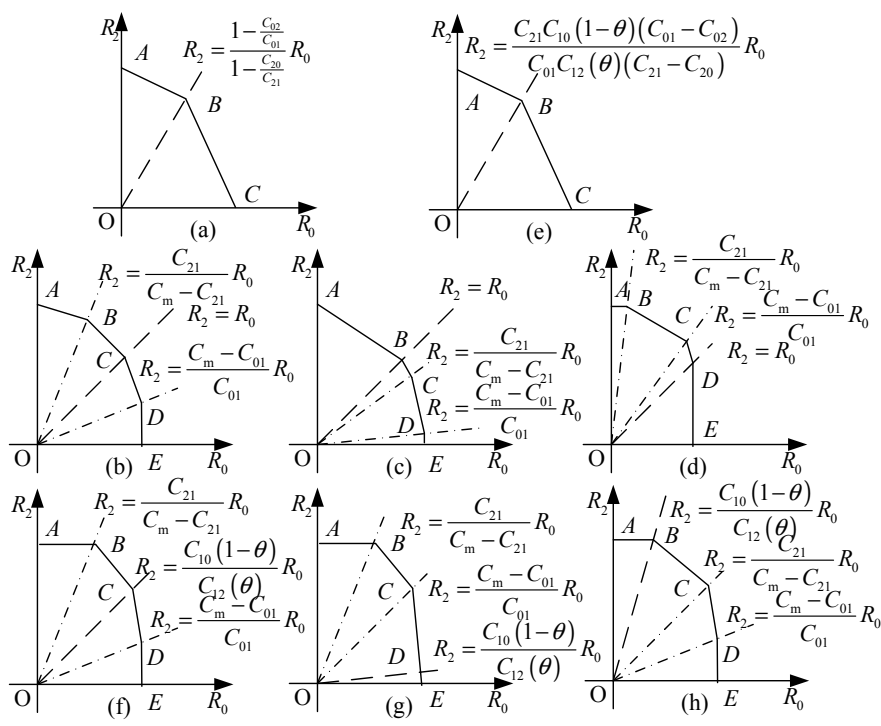

Fig. 1: Rate regions for two-way relaying: 3S-WD-XOR(a) 2S-XOR(b, c, d) 3S-WD-SUP(e) 2S-SUP(f, g, h)

$R_{0}=\left|D_{0}\right| \leq \lambda_{1} C_{01}$ and $R_{2}=\left|D_{2}\right| \leq \lambda_{2} C_{21}$ in order for the relay to decode each packet, where $\lambda_{i}$ is the time fraction occupied by the $i$-step. Due to the presence of direct link, node 2 is able to exact $\left|D_{02}\right| \leq \lambda_{1} C_{02}$ bit information from the node 0 in the fist step, and node 0 to extract $\left|D_{20}\right| \leq \lambda_{2} C_{20}$ bit information from the node 2 in the second step. Thus, in the third step, the relay node only needs to transmit $\left|D_{0}\right|-\left|D_{02}\right|$ bits to node 2 and $\left|D_{2}\right|-\left|D_{20}\right|$ bits to node 0 . We denote the corresponding packets as $D_{0}^{\prime}$ and $D_{2}^{\prime}$. Similar to [11], the relay needs to compare the packet sizes $\left|D_{0}^{\prime}\right|$ and $\left|D_{2}^{\prime}\right|$. Two cases can occur:

1) Case 1: $\left|D_{2}^{\prime}\right| \geq\left|D_{0}^{\prime}\right|$. The relay node 1 partitions the packet $D_{2}^{\prime}$ into $D_{2}^{\prime(1)}$ and $D_{2}^{\prime(2)}$ so that $\left|D_{2}^{\prime(1)}\right|=\left|D_{0}^{\prime}\right|$ and $\left|D_{2}^{\prime(2)}\right|=\left|D_{2}^{\prime}\right|-\left|D_{0}^{\prime}\right|$. Packet $D_{2}^{\prime(1)}$ contains the first $\left|D_{0}^{\prime}\right|$ bits from $D_{2}^{\prime}$ and packet $D_{2}^{\prime(2)}$ contains the rest of the bits from $D_{2}^{\prime}$. Now, node 1 creates $D_{1}^{\prime}=D_{2}^{\prime(1)} \oplus D_{0}^{\prime}$, where $\oplus$ is the bitwise XOR operation. Due to the assumption that $C_{10} \geq C_{12}$ and the condition that both nodes 0 and 2 need to receive it successfully, the time $\lambda_{3}$ duration should be divided two sub-time slots $\lambda_{3^{1}}$ and $\lambda_{3^{2}}$ with $\lambda_{3}=\lambda_{3^{1}}+\lambda_{3^{2}}$. So, the packet $D_{1}^{\prime}$ is transmitted at the lower rate $C_{12}$ for a fraction $\lambda_{3^{1}}$ of time. Thus, we have $R_{0}^{\prime}=\left|D_{0}\right|-\left|D_{02}\right|=\left|D_{1}^{\prime}\right| \leq$ $\lambda_{3^{1}} C_{12}$. After receiving $D_{1}^{\prime}$, node 2 extracts the packet $D_{0}^{\prime}$ as $D_{0}^{\prime}=D_{1}^{\prime} \oplus D_{2}^{\prime(1)}$. Similarly, after receiving $D_{1}^{\prime}$, node 0 extracts $D_{2}^{\prime(1)}=D_{1}^{\prime} \oplus D_{0}^{\prime}$. Next, node 1 transmits the packet $D_{2}^{\prime(2)}$ to node 0 . Since only node 0 needs to decode it, the packet can be transmitted at a higher rate of $C_{10}$ for $\lambda_{3^{2}}$ duration. Therefore, we have $R_{2}^{\prime}-R_{0}^{\prime}=\left(\left|D_{2}\right|-\left|D_{20}\right|\right)-\left(\left|D_{0}\right|-\left|D_{02}\right|\right)=\left|D_{2}^{\prime(2)}\right| \leq$ $\lambda_{3^{2}} C_{10}$. With $D_{2}^{\prime(1)}$ and $D_{2}^{\prime(2)}$, node 0 can now recover $D_{2}^{\prime}$. Finally, since the total time duration is normalized to 1 , we have $\sum_{i=1}^{3} \lambda_{i}=1$.

2) Case 2: $\left|D_{2}^{\prime}\right| \leq\left|D_{0}^{\prime}\right|$. The packet $D_{2}^{\prime}$ is padded with zeros to obtain the packet $D_{2}^{\prime p}$ such that $\left|D_{2}^{\prime p}\right|=\left|D_{0}^{\prime}\right|$. Since node 0 and 2 know the size of $D_{2}^{\prime}$, they also know how many zeros are used for padding. Node 1 creates the packet $D_{1}^{\prime}=D_{2}^{\prime p} \oplus D_{0}^{\prime}$. 
In Step 3 the packet $D_{1}^{\prime}$ is broadcast at a rate at which both node 0 and node 2 can successfully decode. Thus, we have $R_{0}^{\prime}=\left|D_{0}\right|-\left|D_{02}\right|=\left|D_{1}^{\prime}\right| \leq \lambda_{3} C_{12}$, where $\lambda_{3}$ is the time fraction entirely spent on the last step. Node 2 then extracts $D_{0}^{\prime}$ as $D_{0}^{\prime}=D_{2}^{\prime p} \oplus D_{1}^{\prime}$, which is the desired packet sent from node 0 . Similarly, node 0 can obtain $D_{2}^{\prime p}$ from $D_{1}^{\prime}$. The packet $D_{2}^{\prime}$ is then obtained by removing the padding zeros from $D_{2}^{\prime p}$. Again, we also have $\sum_{i=1}^{3} \lambda_{i}=1$ due to the total time constraint.

Note that, for each destination, say node 2 , to recover the desired packet $D_{0}$ from $D_{02}$ and $D_{0}^{\prime}$, a coding method for Gaussian parallel channel should be employed [15].

With the above constraints, we obtain the following linear inequalities about $R_{0}$ and $R_{2}$ after simple manipulation as

$$
\begin{aligned}
R_{0}\left(\frac{1}{C_{01}}+\right. & \left.\frac{C_{01}-C_{02}}{C_{01} C_{12}}-\frac{C_{01}-C_{02}}{C_{01} C_{10}}\right) \\
& +R_{2}\left(\frac{1}{C_{21}}+\frac{C_{21}-C_{20}}{C_{21} C_{10}}\right) \leq 1, \\
R_{0}\left(\frac{1}{C_{01}}+\right. & \left.\frac{C_{01}-C_{02}}{C_{01} C_{12}}\right)+R_{2}\left(\frac{1}{C_{21}}\right) \leq 1 .
\end{aligned}
$$

The rate region of the scheme $3 \mathrm{~S}-\mathrm{WD}-\mathrm{XOR}$ is thus given by the set of all rate pairs that satisfy the two inequalities simultaneously. By graphing the feasible set and considering that $R_{0} \geq 0$ and $R_{2} \geq 0$, a quadrilateral region is obtained. The corners of the region are as given in the Theorem 3.1.

Since the rate region of $3 \mathrm{~S}-\mathrm{WD}-\mathrm{XOR}$ is bounded by linear constraints, the maximum sum-rate $R_{0}+R_{2}$ occurs at one of the vertex.

Remark: Let $C_{02}=C_{20}=0$ in Theorem 3.1, we can obtain the rate region for $3 \mathrm{~S}-\mathrm{ND}-\mathrm{XOR}$. Note that a similar rate region of 3S-ND-XOR is studied in [12]. However, the work in [12] did not consider the packet splitting. Thus, its rate region is only a subset of ours.

Theorem 3.2 (2S-XOR): If $C_{01} \geq(1 / 2) C_{\mathrm{m}}$ and $C_{21} \geq$ $(1 / 2) C_{\mathrm{m}}$, the rate region for $2 \mathrm{~S}-\mathrm{XOR}$ is the hexagon formed by the origin, point A with coordinate $\left(0,\left(1 / C_{21}+1 / C_{10}\right)^{-1}\right)$, B with $\left(\left(\left(C_{21} /\left(C_{\mathrm{m}}-C_{21}\right)\right)\left(1 / C_{21}+1 / C_{10}\right)+1 / C_{12}-\right.\right.$ $\left.1 / C_{10}\right)^{-1},\left(1 / C_{21}+1 / C_{10}+\left(\left(C_{\mathrm{m}}-C_{21}\right) / C_{21}\right)\left(1 / C_{12}-\right.\right.$ $\left.\left.\left.1 / C_{10}\right)\right)^{-1}\right)$, C with $\left(\left(2 / C_{\mathrm{m}}+1 / C_{12}\right)^{-1},\left(2 / C_{\mathrm{m}}+1 / C_{12}\right)^{-1}\right)$, D with $\left(\left(1 / C_{01}+1 / C_{12}\right)^{-1},\left(\left(C_{01} /\left(C_{\mathrm{m}}-C_{01}\right)\right)\left(1 / C_{01}+\right.\right.\right.$ $\left.\left.\left.1 / C_{12}\right)\right)^{-1}\right)$, and $\mathrm{E}$ with $\left(\left(1 / C_{01}+1 / C_{12}\right)^{-1}, 0\right)$, as sketched in Fig. 1(b).

If $C_{01} \geq(1 / 2) C_{\mathrm{m}}$ and $C_{21} \leq(1 / 2) C_{\mathrm{m}}$, the rate region for $2 \mathrm{~S}-\mathrm{XOR}$ is the hexagon formed by the origin, point $\mathrm{A}$ with coordinate $\left(0,\left(1 / C_{21}+1 / C_{10}\right)^{-1}\right)$, B with $\left(\left(1 / C_{21}+\right.\right.$ $\left.\left.1 / C_{12}\right)^{-1},\left(1 / C_{21}+1 / C_{12}\right)^{-1}\right), \mathrm{C}$ with $\left(\left(1 /\left(C_{\mathrm{m}}-C_{21}\right)+\right.\right.$ $\left.\left.1 / C_{12}\right)^{-1},\left(\left(\left(C_{\mathrm{m}}-C_{21}\right) / C_{21}\right)\left(1 /\left(C_{\mathrm{m}}-C_{21}\right)+1 / C_{12}\right)\right)^{-1}\right)$, D with $\left(\left(1 / C_{01}+1 / C_{12}\right)^{-1},\left(\left(C_{01} /\left(C_{\mathrm{m}}-C_{01}\right)\right)\left(1 / C_{01}+\right.\right.\right.$ $\left.\left.\left.1 / C_{12}\right)\right)^{-1}\right)$, and $\mathrm{E}$ with $\left(\left(1 / C_{01}+1 / C_{12}\right)^{-1}, 0\right)$, as sketched in Fig. 1(c).

If $C_{01} \leq(1 / 2) C_{\mathrm{m}}$ and $C_{21} \geq(1 / 2) C_{\mathrm{m}}$, the rate region for $2 \mathrm{~S}-\mathrm{XOR}$ is the hexagon formed by the origin, point $\mathrm{A}$ with coordinate $\left(0,\left(1 / C_{21}+1 / C_{10}\right)^{-1}\right)$, B with $\left(\left(\left(C_{21} /\left(C_{\mathrm{m}}-\right.\right.\right.\right.$ $\left.\left.\left.C_{21}\right)\right)\left(1 / C_{21}+1 / C_{10}\right)+1 / C_{12}-1 / C_{10}\right)^{-1},\left(1 / C_{21}+\right.$ $\left.\left.1 / C_{10}+\left(\left(C_{\mathrm{m}}-C_{21}\right) / C_{21}\right)\left(1 / C_{12}-1 / C_{10}\right)\right)^{-1}\right), \mathrm{C}$ with $\left(\left(1 / C_{01}+1 / C_{12}+\left(C_{\mathrm{m}}-2 C_{01}\right) /\left(C_{01} C_{10}\right)\right)^{-1},\left(\left(C_{01} /\left(C_{\mathrm{m}}-\right.\right.\right.\right.$ $\left.\left.\left.\left.C_{01}\right)\right)\left(1 / C_{01}+1 / C_{12}+\left(C_{\mathrm{m}}-2 C_{01}\right) /\left(C_{01} C_{10}\right)\right)\right)^{-1}\right), \mathrm{D}$ with $\left(\left(1 / C_{01}+1 / C_{12}\right)^{-1},\left(1 / C_{01}+1 / C_{12}\right)^{-1}\right)$, and $\mathrm{E}$ with $\left(\left(1 / C_{01}+1 / C_{12}\right)^{-1}, 0\right)$, as sketched in Fig. 1(d).

Proof: The proof of this theorem differs from the proof of Theorem 3.1 mainly in the multiple-access phase. Due to the constraint of MAC, we have $R_{0} \leq \lambda_{1} C_{01}, R_{2} \leq \lambda_{1} C_{21}$ and $R_{0}+R_{2} \leq \lambda_{1} C_{\mathrm{m}}$ in the first step with time duration $\lambda_{1}$. In the second step with time duration $\lambda_{2}$, we have $R_{0} \leq$ $\lambda_{2^{1}} C_{12}$ and $R_{2}-R_{0} \leq \lambda_{2^{2}} C_{10}$ with $\lambda_{2^{1}}+\lambda_{2^{2}}=\lambda_{2}$, when $C_{01} \geq(1 / 2) C_{\mathrm{m}}, C_{21} \geq(1 / 2) C_{\mathrm{m}}$ and $R_{2} \geq R_{0}$. Note that the condition, $R_{0} \leq \lambda_{1} C_{01}$ can be absorbed from $R_{0}+R_{2} \leq$ $\lambda_{1} C_{\mathrm{m}}, R_{2} \geq R_{0}$ and $C_{01} \geq(1 / 2) C_{\mathrm{m}}$. Then, combining these, we obtain two inequalities as shown below in (2a) and (2b). For the same reason, we also obtain two inequalities (2c) and (2d) when $C_{01} \geq(1 / 2) C_{\mathrm{m}}, C_{21} \geq(1 / 2) C_{\mathrm{m}}$ and $R_{2} \leq R_{0}$.

$$
\begin{aligned}
& R_{0}\left(\frac{1}{C_{12}}-\frac{1}{C_{10}}\right)+R_{2}\left(\frac{1}{C_{21}}+\frac{1}{C_{10}}\right) \leq 1, \\
& R_{0}\left(\frac{1}{C_{\mathrm{m}}}+\frac{1}{C_{12}}-\frac{1}{C_{10}}\right)+R_{2}\left(\frac{1}{C_{\mathrm{m}}}+\frac{1}{C_{10}}\right) \leq 1, \\
& R_{0}\left(\frac{1}{C_{\mathrm{m}}}+\frac{1}{C_{12}}\right)+R_{2}\left(\frac{1}{C_{\mathrm{m}}}\right) \leq 1, \\
& R_{0}\left(\frac{1}{C_{01}}+\frac{1}{C_{12}}\right) \leq 1 .
\end{aligned}
$$

If $C_{01} \geq(1 / 2) C_{\mathrm{m}}$ and $C_{21} \leq(1 / 2) C_{\mathrm{m}}$, the above inequalities remain except that $(2 \mathrm{~b})$ should be changed to

$$
R_{0}\left(\frac{1}{C_{12}}\right)+R_{2}\left(\frac{1}{C_{21}}\right) \leq 1 .
$$

Otherwise, if $C_{01} \leq(1 / 2) C_{\mathrm{m}}$ and $C_{21} \geq(1 / 2) C_{\mathrm{m}}$, (2c) should be changed to

$$
R_{0}\left(\frac{1}{C_{01}}+\frac{1}{C_{12}}-\frac{1}{C_{10}}\right)+R_{2}\left(\frac{1}{C_{10}}\right) \leq 1 .
$$

Note the condition that both $C_{01}$ and $C_{21}$ are less than $(1 / 2) C_{\mathrm{m}}$ does not exist. Hence, the rate region stated in Theorem 3.2 is proved.

Note that authors in [11] also studied the rate pair of $2 S$ $\mathrm{XOR}$, but only the maximum sum-rate is considered and no rate region is discussed. In addition, the work in [11] is only suitable for symmetric channels with $C_{01}=C_{10}, C_{21}=C_{12}$.

\section{B. Achievable rate region for superposition based DF}

In superposition-based $\mathrm{DF}$, we let $\theta$ present the power ratio allocated to the signal $\mathbf{x}_{0}$ to be transmitted to node 2 and $1-\theta$ be the power ratio on the signal $\mathbf{x}_{2}$ to node 0 , where $0 \leq \theta \leq$ 1. Then the channel capacity of the link $1 \rightarrow 2$ and link $1 \rightarrow 0$ becomes $C_{12}(\theta)=C\left(\theta \gamma_{12}\right)$ and $C_{10}(1-\theta)=C\left((1-\theta) \gamma_{10}\right)$, respectively.

Theorem 3.3 (3S-WD-SUP): The rate region for 3SWD-SUP is the union of the quadrilaterals formed by the origin, point $\mathrm{A}$ with coordinate $\left(0,\left(1 / C_{21}+\left(\left(C_{21}-\right.\right.\right.\right.$ $\left.\left.\left.\left.C_{20}\right) / C_{21}\right)\left(1 / C_{10}(1-\theta)\right)\right)^{-1}\right)$, B with $\left(\left(1 / C_{01}+1 / C_{12}(\theta)-\right.\right.$ $C_{02} /\left(C_{01} C_{12}(\theta)\right)+C_{10}(1-\theta)\left(C_{01}-C_{02}\right) /\left(C_{01} C_{12}(\theta)\left(C_{21}-\right.\right.$ $\left.\left.\left.C_{20}\right)\right)\right)^{-1},\left(1 / C_{21}+1 / C_{10}(1-\theta)-C_{20} /\left(C_{21} C_{10}(1-\theta)\right)+\right.$ $\left.\left.C_{12}(\theta)\left(C_{21}-C_{20}\right) /\left(C_{21} C_{10}(1-\theta)\left(C_{01}-C_{02}\right)\right)\right)^{-1}\right)$, and $\mathrm{C}$ 
with $\left(\left(1 / C_{01}+\left(\left(C_{01}-C_{02}\right) / C_{21}\right)\left(1 / C_{12}(\theta)\right)\right)^{-1}, 0\right)$, for all possible $\theta \in[0,1]$, as sketched in Fig. 1(e).

Proof: In the considered superposition-based DF, the information bits from the two source nodes are encoded separately into two codewords with the same length, which are then superimposed together in the complex field. Unlike XOR-based DF, there is no need to compare the packet sizes of $D_{0}$ and $D_{2}$. It becomes clear that the rate pair $\left(R_{0}, R_{2}\right)$ has to satisfy the following conditions: $R_{0} \leq \lambda_{1} C_{01}, R_{2} \leq \lambda_{2} C_{21}$, $R_{0}^{\prime} \leq \lambda_{3} C_{12}(\theta)$ and $R_{2}^{\prime} \leq \lambda_{3} C_{10}(1-\theta)$ with $\sum_{i=1}^{3} \lambda_{i} \leq 1$. Here, $R_{0}^{\prime}$ and $R_{2}^{\prime}$ are defined in a similar way as in the proof of Theorem 3.1 (3S-WD-XOR) and given by $R_{0}^{\prime}=R_{0}-\lambda_{1} C_{02}$ and $R_{2}^{\prime}=R_{2}-\lambda_{2} C_{20}$. Eliminating the time variables $\lambda_{i}$ 's, we obtain the linear inequalities about $R_{0}$ and $R_{2}$ as

$$
\begin{aligned}
& R_{0}\left(\frac{1}{C_{01}}\right)+R_{2}\left(\frac{1}{C_{21}}+\frac{C_{21}-C_{20}}{C_{21} C_{10}(1-\theta)}\right) \leq 1, \\
& R_{0}\left(\frac{1}{C_{01}}+\frac{C_{01}-C_{02}}{C_{01} C_{12}(\theta)}\right)+R_{2}\left(\frac{1}{C_{21}}\right) \leq 1 .
\end{aligned}
$$

Since the power ratio $\theta$ can take any value from 0 to 1 , the rate region of this $3 \mathrm{~S}-\mathrm{WD}$-SUP scheme is formed by the union of all sets satisfying the above inequalities for every possible $\theta \in[0,1]$. The theorem is thus proved.

Remark: Let $C_{02}=C_{20}=0$ in the Theorem 3.3, we can obtain the rate region for $3 \mathrm{~S}-\mathrm{ND}-\mathrm{SUP}$.

Theorem 3.4 (2S-SUP): If $\left(C_{\mathrm{m}}-C_{01}\right) / C_{01} \leq C_{10}(1-$ $\theta) / C_{12}(\theta) \leq C_{21} /\left(C_{\mathrm{m}}-C_{21}\right)$, the rate region for $2 \mathrm{~S}$ SUP is the union of the hexagons formed by the origin, point $\mathrm{A}$ with coordinate $\left(0,\left(1 / C_{21}+1 / C_{10}(1-\right.\right.$ $\left.\theta))^{-1}\right)$, B with $\left(\left(\left(C_{21} /\left(C_{\mathrm{m}}-C_{21}\right)\right)\left(1 / C_{21}+1 / C_{10}(1-\right.\right.\right.$ $\left.\theta)))^{-1},\left(1 / C_{21}+1 / C_{10}(1-\theta)\right)^{-1}\right), \mathrm{C}$ with $\left(\left(1 / C_{\mathrm{m}}+\right.\right.$ $\left.1 / C_{12}(\theta)+C_{10}(1-\theta) /\left(C_{\mathrm{m}} C_{12}(\theta)\right)\right)^{-1},\left(1 / C_{\mathrm{m}}+1 / C_{10}(1-\right.$ $\left.\left.\theta)+C_{12}(\theta) /\left(C_{\mathrm{m}} C_{10}(1-\theta)\right)\right)^{-1}\right), \mathrm{D}$ with $\left(\left(1 / C_{01}+\right.\right.$ $\left.\left.1 / C_{12}(\theta)\right)^{-1},\left(\left(C_{01} /\left(C_{\mathrm{m}}-C_{01}\right)\right)\left(1 / C_{01}+1 / C_{12}(\theta)\right)\right)^{-1}\right)$, and $\mathrm{E}$ with $\left(\left(1 / C_{01}+1 / C_{12}(\theta)\right)^{-1}, 0\right)$, for all $\theta \in[0,1]$, as sketched in Fig. 1(f).

If $C_{10}(1-\theta) / C_{12}(\theta) \leq\left(C_{\mathrm{m}}-C_{01}\right) / C_{01}$, the rate region for 2S-SUP is the union of the hexagons formed by the origin, point A with coordinate $\left(0,\left(1 / C_{21}+1 / C_{10}(1-\theta)\right)^{-1}\right)$, B with $\left(\left(\left(C_{21} /\left(C_{\mathrm{m}}-C_{21}\right)\right)\left(1 / C_{21}+1 / C_{10}(1-\theta)\right)\right)^{-1},\left(1 / C_{21}+\right.\right.$ $\left.\left.1 / C_{10}(1-\theta)\right)^{-1}\right)$, C with $\left(\left(\left(C_{\mathrm{m}}-C_{01}\right) / C_{01}\right)\left(1 /\left(C_{\mathrm{m}}-\right.\right.\right.$ $\left.\left.\left.\left.C_{01}\right)+1 / C_{10}(1-\theta)\right)\right)^{-1},\left(1 /\left(C_{\mathrm{m}}-C_{01}\right)+1 / C_{10}(1-\theta)\right)^{-1}\right), \mathrm{D}$ with $\left(\left(1 / C_{01}+1 / C_{12}(\theta)\right)^{-1},\left(\left(C_{12}(\theta) / C_{10}(1-\theta)\right)\left(1 / C_{01}+\right.\right.\right.$ $\left.\left.\left.1 / C_{12}(\theta)\right)\right)^{-1}\right)$ and $\mathrm{E}$ with $\left(\left(1 / C_{01}+1 / C_{12}(\theta)\right)^{-1}, 0\right)$, for all $\theta \in[0,1]$, as sketched in Fig. $1(\mathrm{~g})$.

If $C_{10}(1-\theta) / C_{12}(\theta) \geq C_{21} /\left(C_{\mathrm{m}}-C_{21}\right)$, the rate region for 2S-SUP is the union of the hexagons formed by the origin, point $\mathrm{A}$ with coordinate $\left(0,\left(1 / C_{21}+1 / C_{10}(1-\right.\right.$ $\left.\theta))^{-1}\right)$, B with $\left(\left(\left(C_{10}(1-\theta) / C_{12}(\theta)\right)\left(1 / C_{21}+1 / C_{10}(1-\right.\right.\right.$ $\left.\theta)))^{-1},\left(1 / C_{21}+1 / C_{10}(1-\theta)\right)^{-1}\right), \quad \mathrm{C} \quad w i t h\left(\left(1 /\left(C_{\mathrm{m}}-\right.\right.\right.$ $\left.\left.C_{21}\right)+1 / C_{12}(\theta)\right)^{-1},\left(\left(\left(C_{\mathrm{m}}-C_{21}\right) / C_{21}\right)\left(1 /\left(C_{\mathrm{m}}-C_{21}\right)+\right.\right.$ $\left.\left.\left.1 / C_{12}(\theta)\right)\right)^{-1}\right)$, D with $\left(\left(1 / C_{01}+1 / C_{12}(\theta)\right)^{-1},\left(\left(C_{01} /\left(C_{\mathrm{m}}-\right.\right.\right.\right.$ $\left.\left.\left.C_{01}\right)\left(1 / C_{01}+1 / C_{12}(\theta)\right)\right)^{-1}\right)$, and $\mathrm{E}$ with $\left(\left(1 / C_{01}+\right.\right.$ $\left.\left.1 / C_{12}(\theta)\right)^{-1}, 0\right)$, for all $\theta \in[0,1]$, as sketched in Fig. 1(h).

Proof: Using similar arguments as in the proofs of Theorem 3.2 (2S-XOR) and Theorem 3.3 (3S-WD-SUP), we obtain the linear inequalities that the rate pair $\left(R_{0}, R_{2}\right)$ must satisfy

$$
\begin{aligned}
& R_{2}\left(\frac{1}{C_{21}}+\frac{1}{C_{10}(1-\theta)}\right) \leq 1 \\
& R_{0}\left(\frac{1}{C_{\mathrm{m}}}\right)+R_{2}\left(\frac{1}{C_{\mathrm{m}}}+\frac{1}{C_{10}(1-\theta)}\right) \leq 1, \\
& R_{0}\left(\frac{1}{C_{\mathrm{m}}}+\frac{1}{C_{12}(\theta)}\right)+R_{2}\left(\frac{1}{C_{\mathrm{m}}}\right) \leq 1, \\
& R_{0}\left(\frac{1}{C_{01}}+\frac{1}{C_{12}(\theta)}\right) \leq 1
\end{aligned}
$$

for $\left(C_{\mathrm{m}}-C_{01}\right) / C_{01} \leq C_{10}(1-\theta) / C_{12}(\theta) \leq C_{21} /\left(C_{\mathrm{m}}-C_{21}\right)$. If $C_{10}(1-\theta) / C_{12}(\theta) \geq C_{21} /\left(C_{\mathrm{m}}-C_{21}\right)$, the same set applies except that (4b) should be changed to

$$
R_{0}\left(\frac{1}{C_{12}(\theta)}\right)+R_{2}\left(\frac{1}{C_{21}}\right) \leq 1 .
$$

Else, if $C_{10}(1-\theta) / C_{12}(\theta) \leq\left(C_{\mathrm{m}}-C_{01}\right) / C_{01}$, (4c) should be changed to

$$
R_{0}\left(\frac{1}{C_{01}}\right)+R_{2}\left(\frac{1}{C_{10}(1-\theta)}\right) \leq 1
$$

The theorem is thus proved.

The rate pair of 2S-SUP is also studied in [8], [13]. However, the authors did not derive the explicit closed-form expressions.

\section{NUMERAL RESULTS AND DISCUSSIONS}

Suppose that the channel gain on each link is modeled by the distance path loss model, given by $\alpha_{i j}=c \cdot d_{i j}^{-n}$, where $c$ is an attenuation constant, $n$ is the path loss exponent and fixed at 3 throughout this section, and $d_{i j}$ denotes the distance between nodes $i$ and $j$. For simplicity, each node uses the same transmission power. The SNR on the direct link is fixed at $\gamma_{02}=\gamma_{20}=0 \mathrm{~dB}$. We consider the network layout, where the distance between nodes 0 and 2 is normalized to 1 and the location of the relay is determined using the projections $x$ and $y$. The source nodes 0 and 2 have the coordinates $(-0.5,0)$ and $(0.5,0)$, respectively. And for any relay on the plane with coordinate $(x, y)$, we can calculate $d_{01}=\sqrt{(x+0.5)^{2}+y^{2}}$, and $d_{12}=\sqrt{(x-0.5)^{2}+y^{2}}$. For comparison purpose, the optimal rate regions of the 3-step scheme with direct link and 2-step scheme using random binning derived in [9], [10] are also shown and denoted as "3S-WD-Capa" and "2SCapa", respectively. Moreover, the 2-step point to point (2SPTP) direct communication is also considered as a benchmark scheme.

From Fig. 2, it is observed that when the relay lies in the middle, the XOR schemes are capacity achieving whereas the SUP schemes are much inferior. But this is not the case when the relay is more closer to node 0 as shown in Fig. 2(b). Fig. 2 also shows that 3-step schemes can achieve higher oneway transmission rate than 2-step schemes. This is expected because 3-step schemes exploit the direct link. However if the direct link is absent, 3-step schemes become inferior to their 2step counterparts (Numerical results are skipped due to space limitation). 


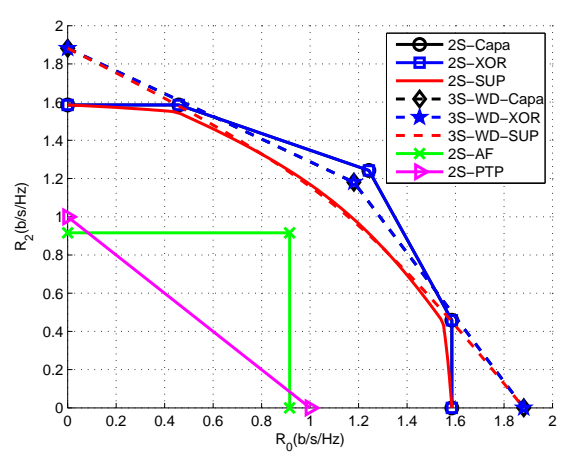

(a) $x=y=0$

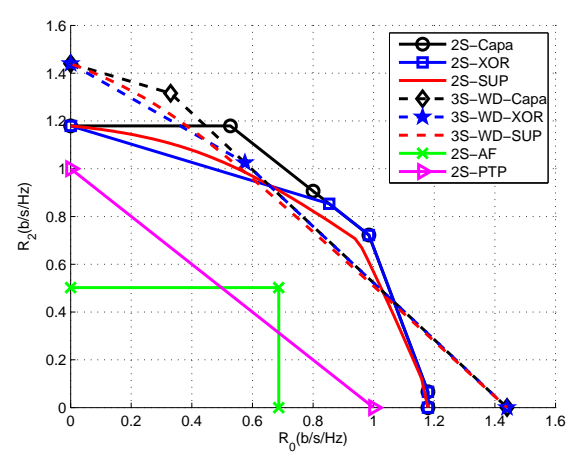

(b) $x=-0.2, y=0.3$

Fig. 2: Rate region $R_{2}$ versus $R_{0}$ for two-way relaying

Fig. 3 shows the maximum sum-rates achieved by different relay schemes. In Fig. 3(a), no matter where the relay is, 2S-XOR always achieves the largest sum-rate among the considered schemes. From Fig. 3(b), we see that XOR schemes always achieve larger sum-rates than SUP schemes under the same condition. In the region $y \leq 0.6,2 \mathrm{~S}-\mathrm{XOR}$ is the optimal. As $y$ approach $\sqrt{3} / 2\left(d_{01}=d_{12}\right.$ approach 1$)$, 3S-WD-XOR becomes the best, and 3S-WD-XOR/SUP all approach the sum-rate of 2S-PTP, while 2S-AF becomes worst.

\section{CONClusion}

We characterized the achievable rate regions for a few two-way relay schemes, including 3S-ND-XOR/SUP, 3S-WD$\mathrm{XOR} / \mathrm{SUP}$ and 2S-XOR/SUP. Each of them is expressed analytically together with a graphical sketch. These results are suitable for any Gaussian channel with fixed channel gain. Our analysis shows that XOR-based DF is capacity achieving in symmetrical relay channels while superpositionbased DF can achieve rate region where XOR-based DF cannot in asymmetrical relay channels. Among these schemes, AF is the worst.

\section{REFERENCES}

[1] R. Ahlswede, N. Cai, S.-Y. R. Li, and R. W. Yeung, "Network information flow," IEEE Trans. Inform. Theory, vol. 46, no. 4, pp. 1204-1216, July 2000.

[2] Y. Wu, P. A. Chou, and S.-Y. Kung, "Information exchange in wireless networks with network coding and physical-layer broadcast," in Proc. 39th Conf. on Inform. Sciences and Systems (CISS), Mar. 2005.

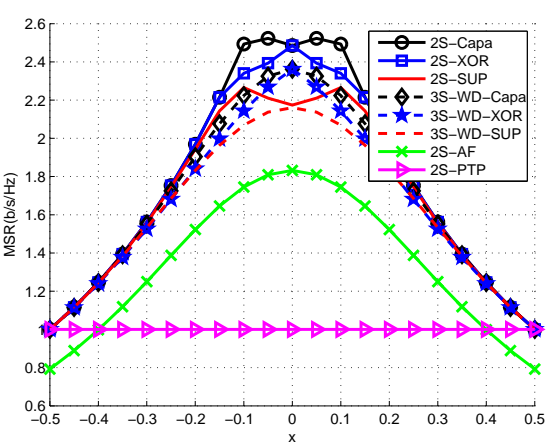

(a) $-0.5 \leq x \leq 0.5, y=0$

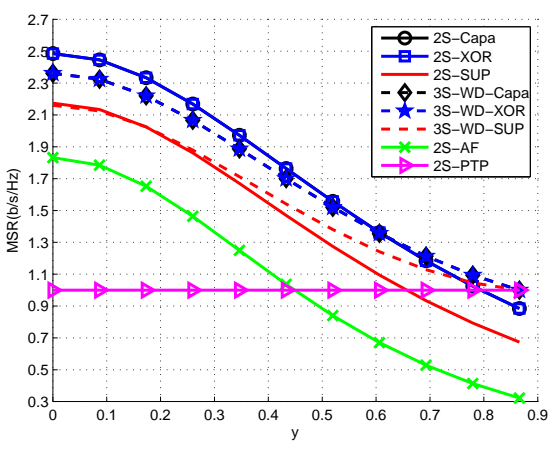

(b) $x=0,0 \leq y \leq \frac{\sqrt{3}}{2}$

Fig. 3: Maximum sum-rate versus distance for two-way relaying

[3] P. Larsson, N. Johansson, and K.-E. Sunell, "Coded bi-directional relaying," in Proc. 5th Scandinavian Workshop on Ad Hoc Networks (ADHOC05), May 2005.

[4] P. Popovski and H. Yomo, "Bi-directional amplification of throughput in a wireless multi-hop network," in Proc. IEEE Veh. Tech. Conf. (VTC), May 2006.

[5] C. Hausl and J. Hagenauer, "Iterative network and channel decoding for the two-way relay channel," in Proc. IEEE Int. Conf. on Comm. (ICC), Jun. 2006.

[6] S. Katti, H. Rahul, W. Hu, D. Katabi, M. M. edard, and J. Crowcroft, "XORs in the air: Practical wireless network coding," in Proc. ACM SIGCOMM Conf., Sept. 2006.

[7] S. Zhang, S. C. Liew, and P. P. Lam, "Physical-layer network coding," in Proc. ACM SIGCOMM Conf., Sept. 2006.

[8] B. Rankov and A. Wittneben, "Spectral efficient protocols for halfduplex fading relay channels," IEEE Jour. Selec. Areas. Comm., vol. 25, no. 2, pp. 379-389, Feb. 2007.

[9] T. J. Oechtering, C. Schnurr, I. Bjelakovic, and H. Boche, "Broadcast capacity region of two-phase bidirectional relaying," IEEE Trans. Inform. Theory, vol. 54, no. 1, pp. 454-458, Jan. 2008.

[10] L. L. Xie, "Network coding and random binning for multi-user channels," in Proc. IEEE 10th Canadian Workshop on Inform. Theory, June 2007, pp. 85-88.

[11] P. Popovski and H. Yomo, "Physical network coding in two-way wireless relay channels," in Proc. IEEE Int. Conf. on Comm. (ICC), June 2007, pp. $707-712$.

[12] C. H. Liu and F. Xue, "Network coding for two-way relaying: rate region, sum rate and opportunistic scheduling," in Proc. IEEE Int. Conf. on Comm. (ICC), May 2008, pp. 1044-1049.

[13] T. J. Oechtering and H. Boche, "Stability region of an optimized bidirectional regenerative half-duplex relaying protocol," IEEE Trans. Сотти., vol. 56, no. 9, pp. 1519-1529, Sep. 2008.

[14] R. F. Wyrembelski, T. J. Oechtering, and H. Boche, "Decode-andforward strategies for bidirectional relaying," in Proc. IEEE Int. Symp. on Indoor and Mobile Radio Comm. (PIMRC), Sept. 2008, pp. 1-6.

[15] A. H.-Madsen, "Capacity bounds for cooperative diversity," IEEE Trans. Inform. Theory, vol. 52, no. 4, pp. 1522-1544, April 2006. 